\title{
Computer Network Security Evaluation Based on Fuzzy Analytic Hierarchy Process
}

\author{
Jianhang Wang ${ }^{1, \text { a }}$, Haibo Wang ${ }^{2}$ \\ ${ }^{1}$ Jilin Polytechnic of Communications, Jilin Changchun, China, 130012 \\ ${ }^{2}$ Changchun Chengyuan Tech Bussiness Co.,Ltd. \\ acmz997@sina.com
}

Keywords: Computer Network Security Evaluation, Fuzzy Analytic Hierarchy Process

\begin{abstract}
With the rapid development and application of computer network technology, its classical linear evaluation method has been unable to meet the needs of people's computer network security evaluation because of its shortcomings such as low evaluation accuracy. Therefore, the article explores the higher precision fuzzy analytic hierarchy process. The application of computer network security is in order to solve the security risks of computer network systems and other issues.
\end{abstract}

\section{Introduction}

Computer network security evaluation is a basic work to determine the security performance of network systems and implement network security management. It involves many factors such as organizational management, network technology, personnel psychology and social environment. In recent years, with the rapid development of information technology in China, computer network security issues have become increasingly prominent. Although domestic and foreign scholars have used different methods to seek effective methods for network security evaluation from different aspects, many of the network security concerns are involved. The factors are difficult to quantify. So far, there is no mature quantitative evaluation method for network security. Based on the understanding of the factors affecting network security, this paper establishes a complete computer network security evaluation index system. Based on this, a fuzzy analytic Hierarchy Process (FAHP) is used to construct a computer network security evaluation based on triangular fuzzy numbers. The model is verified by examples to find a scientific, effective and feasible computer network security evaluation method.

\section{Introduction to a Fuzzy Analytic Hierarchy Process}

Fuzzy analytic hierarchy process is a product of fuzzy mathematics and analytic hierarchy process. The analytic hierarchy process mainly studies the role of people's ideological judgments in the process of decision-making, that is, transforms people's subjective judgments into mathematical problems, thus helping people solve difficult and more complex decision-making problems and fully quantify the problems. The traditional analytic hierarchy process uses the 1-9 scale method to form a comparative judgment matrix. Although discrete numbers are relatively simple to use, they are not able to better reflect people's judgment ambiguity. The Dutch researchers used the traditional analytic hierarchy process as the research basis, and constructed a new analytic hierarchy process based on it, which specifically refers to the use of triangular fuzzy numbers to form a fuzzy judgment matrix, so as to achieve the purpose of using analytic hierarchy process in a fuzzy environment.

\section{Overview of the Characteristics and Types of Security Risks in Computer Networks}

In recent years, with the continuous development of information technology, computer network 
technology has been integrated into people's daily life, and more and more people pay attention to it. The application of this technology is more and more extensive. Especially in 2016, in the context of the information age, people's daily life is inseparable from computer networks. However, while computer network technology brings convenience to people's lives, it also brings some security risks to people. Computer network security issues are critical. In order to effectively solve this problem, it is necessary to adopt corresponding management methods to ensure that the computer is always in a safe state during the operation process. At the same time, the hardware and software of the computer cannot be infringed, so as to ensure the safe use of the user. Computers can bring convenience to people. The biggest feature is network sharing. People can use the computer network to find the information they need, but at the same time, this convenience also poses a great threat to the computer operating environment. The specific threats include the following three aspects:

During the operation of the computer network, it is vulnerable to some malicious attacks. However, after the computer network system is attacked, it will not be able to operate normally, or even cause the entire system to be paralyzed. Among them, the most common malicious attack is a computer virus, which has the characteristics of infection and latent characteristics. It can lurk the computer in a static state before the computer is turned on, hidden in the storage medium inside the computer, when the computer is turned on. At that moment, the propagating molecules in the computer virus will be activated. If the computer is connected to some mobile devices, then the virus will use the computer as a transmission medium and spread to these mobile devices, causing other programs to be modified. Destroy the purpose of its internal system.

Under normal circumstances, the intrusions and attacks on the computer are somewhat concealed and latent, so when the computer is not working properly, it is difficult to find the problem, which is the result of the characteristics of these destruction factors.

The computer network system will not have any symptoms before it is attacked. Sometimes we see that the computer is running normally, but there is also the possibility of attack. After the computer runs for a while, the system will be paralyzed. , causing it to not function properly. In addition, the potential damage factor of the computer is also diffuse and contagious in the event of abrupt, and the computer network system itself is highly interconnected, and multiple computers are connected together, if one of the computers is exposed to a virus. So, it is very likely that the computer in this computer room will be infected, causing paralysis of all systems.

In summary, with the rapid development of science and technology, although the application scope of computer network technology is more and more extensive, it is accompanied by some security risks, resulting in more hidden dangers, such as computer network technology is not perfect. It lack of a relatively sound security system and insufficient security configuration, so the main way to solve these problems is to improve the prevention technology of computer network security.

Computer network security risks mainly include the following three types: password intrusion. During the operation of the computer network, some illegal intruders use the account login or theft of the user's password to cause damage to the environment in which the computer network operates. After the user's account password is cracked, the illegal intruder will log in through the legal account. After entering the user's network, attack it. Usually, when users access computer web pages or Web sites, they do not consider security issues, which lead hackers to take advantage of them. They tamper with the URLs visited by users. When users log in to this page again, hackers will adopt security holes. Attack the user's computer system. Email attack. The illegal intruder sends the virus to the user in the form of an email. When the user views the email, the virus invades the user's computer network.

\section{Specific Steps to Implement Fuzzy Analytic Hierarchy Process in Computer Network Security Evaluation}

Computer network security technology plays a vital role in the normal operation of computers, and it can bring together computer information technology and other related disciplines. In the context of the Internet age, the development of many modern technologies is inseparable from 
computer networks. Through the computer network platform, interoperability between various fields can be realized, thus promoting the development speed of the field. Therefore, computer network technology is developing for the current era. The role is very large, and people's dependence on the network is getting more and more serious. At present, many industries have already studied computer network technology. Fuzzy analytic hierarchy analysis (AHP) is an important method to evaluate computer network security problems. Its application scope is more and more extensive. The specific implementation steps are as follows:

Before constructing the hierarchical model, it is necessary to understand the nature of the problem and related requirements, and analyze it to make the various influencing factors reasonably divided, and to make different levels of combinations according to the relationship between the various factors. Aggregate to create a scientific and effective hierarchical model.

Construct a fuzzy judgment matrix. The 1-9 scale description is used as the construction basis, and each factor is analyzed by means of pairwise comparison. Firstly, the importance of the upper layer factor is judged, and the result of the judgment is expressed by the triangular fuzzy number, and the judgment result is obtained. As a basis, it lays the foundation for the formation of the fuzzy judgment matrix.

The core step of applying fuzzy AHP to computer network security evaluation is defuzzification. The application object of this operation step is fuzzy judgment matrix. After defuzzification, it can be transformed into non-fuzzy judgment matrix. And the basis of the fuzzy AHP method applied will be set to the non-fuzzy judgment matrix. In order to effectively judge the maximum eigenvalue max corresponding to the defuzzification matrix, it is necessary to select the corresponding factors of the same level as the sorting basis for the importance of the factors of the previous level, so as to achieve a reasonable ordering of the weights of each factor.

The total ordering of the levels is actually to reasonably rank the weights of the target layers of the various schemes of the stratum, and it is necessary to calculate the corresponding weight values, perform hierarchical single ordering in order from top to bottom, and synthesize the results of the single sorting to construct the total ordering of the levels.

\section{Advantages and Applications of Fuzzy Analytic Hierarchy Process in Computer Network Security Evaluation}

Fuzzy analytic hierarchy process (AHP) is a systematic analysis method that is qualitatively and quantitatively combined in the United States in the 1970s. The effective use of this method provides a more reliable basis for the quantitative evaluation indicators and the selection of optimization schemes. The basic idea of fuzzy analytic hierarchy process is: In the fuzzy analytic hierarchy process, two different factors are compared and judged, and the comparison is made by comparing the importance degree of different factors, and then by obtaining a fuzzy judgment matrix one step judgment. Moreover, when using fuzzy level judgment method to make decision-making, it pays more attention to the embodiment of human's ability of thinking and judgment, which is also a guarantee to ensure that the decision-making can be more scientific and effective. By mathematicalizing people's thinking, it helps people to quantify and analyze some decision-making problems that are more complex and difficult to accurately grasp.

Compared with the traditional analysis method, the biggest advantage of the fuzzy analytic hierarchy process is that it can ensure the scientificity of evaluation and judgment when conducting computer network security evaluation, and thus ensure that the created safety evaluation system is more valuable. Moreover, in the process of perfecting the network security system, many factors need to be considered, and each factor should be divided into three levels: the target layer, the criterion layer and the decision layer. In order to ensure the accuracy and consistency of the judgment matrix, it is necessary to test the matrix after fuzzification to ensure the consistency in the implementation process. If the matrix cannot guarantee the consistency, it needs to be further corrected. It should also ensure that the criteria layer is weighted relative to the target layer, and in this process to ensure the security of the system, such as ensuring physical security, control the climate and temperature in the computer room, do fire, lightning and anti-static treatment, stabilize 
Good equipment; ensure logical security, timely backup of various data, and timely complete system security audit, do anti-hacking and anti-virus treatment measures; do security management, establish and improve security management system, and ensure good information security, Take certain measures to complete the anti-theft and anti-lost measures. Finally, in order to ensure the total ordering weight of the solution layer relative to the target layer, the bottom-level sorting weight should also be carefully calculated, and the reasonable selection of the product method should be ensured in the calculation.

\section{Conclusion}

With the continuous improvement of the level of social network informationization, the security of computer networks in use has become more and more important. And doing computer network security evaluation is an important condition to ensure its security. Therefore, in today's computer network technology is so developed, in order to fundamentally eliminate various security risks, it is necessary to propose measures to protect against computer network security, and to ensure the security of the computer network technology while ensuring its own security. Provide more reliable reference materials for peer workers.

\section{References}

[1] Li Fangwei, Yang Shaocheng, Zhu Jiang. An improved network security situation assessment method based on fuzzy hierarchy method [J]. Journal of Computer Applications, 2014, 34(9): 2262-2626.

[2] Fei Jun, Yu Lihua. Computer Network Security Evaluation Based on Fuzzy Analytic Hierarchy Process [J]. Computer Applications and Software, 2011(10): 120-123, 166.

[3] Fei Jun, Yu Lihua. Computer Network Security Evaluation Based on Fuzzy Analytic Hierarchy Process [J]. Computer Applications and Software, 2011(10): 120-123, 166.

[4] Wang Yue. Application Analysis of Fuzzy Analytic Hierarchy Process in Computer Network Security Evaluation [J]. Network security technology and application, 2014(12):68, 71.

[5] Gu Fangyong, Liu Fuqiang. Application of Fuzzy Analytic Hierarchy Process in Computer Network Security Evaluation [J].Information Security and Technology, 2015(7):12-14. 